\title{
Risultati di prove su modelli. Profili di resistività apparente per corpi parallelepipedi resistivi immersi in un mezzo omogeneo isotropo conduttivo
}

\author{
(Results obtained through model tests. Apparent resistivity profiles for \\ resistive parallelepipeda in a homogeneous isotrope and conductive \\ medium burried).
}

E. BRIZZOLARI (*)

Ricevuto il 22 Luglio 1972

Rrassunto. - Sono state realizzate prove su modelli per determinare profili di resistività apparente per corpi parallelepipedi resistivi immersi in un mezzo omogeneo isotropo conduttivo. Sono state fatte variare le profondità e le dimensioni del parallelepipedo (spessore, altezza, larghezza) assumendo come unitaria la distanza elettrodica.

E stato cosi possibile determinare l'influenza delle dimensioni del parallelepipedo e della profondita sull'andamento e sui valori massimi delle anomalie.

SumMaRY. - Through model tests, apparent resistivity profiles for resistive parallelepipeda burried in a homogeneous isotrope medium are determined. Depth and dimentions of the parallelepipedum (thickness, height and width) have been changed assuming as unit the electrode spacing.

Influence of depth and dimentions of the parallelepipedum on the anomalies trend and highest values are ascertained.

\section{Prenesse}

Si è avuta occasione di effettuare una ricerca geoelettrica, con il metodo dei profili di resistività, di masse resistive in forma presumibilmente parallelepipeda, immerse in un mezzo conduttivo.

(*) Assistente Ordinario. Istituto di Geofisica Mineraria. Facoltà di Ingegneria di Roma. 
Per la migliore impostazione dell'indagine occorreva scegliere le dimensioni del dispositivo da impiegare nei profili di resistività previsti per la ricerca. Occorreva inoltre stabilire la distanza tra le misure di resistivitì contigue.

Dovendo tale scelta essere evidentemente fatta sulla base di anomalie teoriche, ¿̀ stata effettuata una ricerca di eventuali stucli sull'argomento. î stato però possibile reperire soltanto alcune informazioni di carattere qualitativo e, solo per alcuni casi particolari, anche valutazioni quantitative delle anomalie $\left({ }^{3}\right)$.

Si ̀̀ quindi iniziato uno studio dell'influenza delle dimensioni e della posizione del corpo rispetto al dispositivo elettrodico sulle anomalie. Poiché lo studio è risultato di interesse generalo vengono riportati nella presente nota i risultati cui si è giunti.

î noto che non è stata ancora trovata una soluzione rigorosa del problema del potenziale dovuto ad un elettrodo puntiforme in presenza di un corpo parallelepipedico di resistività differente del mezzo circostante. Per tale ragione il problema è stato affrontato mediante l'uso di modelli.

Per semplicità ̀̀ stato considerato un corpo a resistività molto maggiore del mezzo circostante $(K=1)$, di forma parallelepipeda posto verticalmente al di sotto di un suolo orizzontale e con la faccia superiore parallela al suolo stesso. it stato scelto il dispositivo elettrodico tipo Schlumberger.

Si sono pertanto ricavate le anomalie in funzione dei diversi parametri che potevano essere fatti variare. Questi parametri sono (fig. 1): la profondità $p$ della superficie superiore del corpo dalla superficie del terreno, lo spessore $s$, l'altezza $h$ e la larghezza $l$ del corpo. La distanza elettrodica $A B$ è stata assunta come unitaria. Sono pertanto 4 i parametri che possono variare indipendentemente l'uno dall'altro per $\infty^{4}$ combinazioni possibili.

Le conseguenze di tali variazioni sulle anomalie non sono in molti casi valutabili intuitivamente. Ad esempio un aumento della distanza elettrodica $A B$ provoca un aumento della profondità di investigazione del dispositivo e quindi, costante la profondità del corpo, si dovrebbe ottenere una esaltazione della anomalia.

D'altra parte però, lo stesso aumento della distanza elettrodica potrebbe incrementare il numero delle linee di corrente che si chiudono al di sotto e lateralmente al corpo resistivo se la sua larghezza e altezza non sono tali da impedirlo.

Un aumento dello spessore, in riferimento alla distanza elettrodica, provoca un aumento di valori massimi dell'anomalia e un allar- 


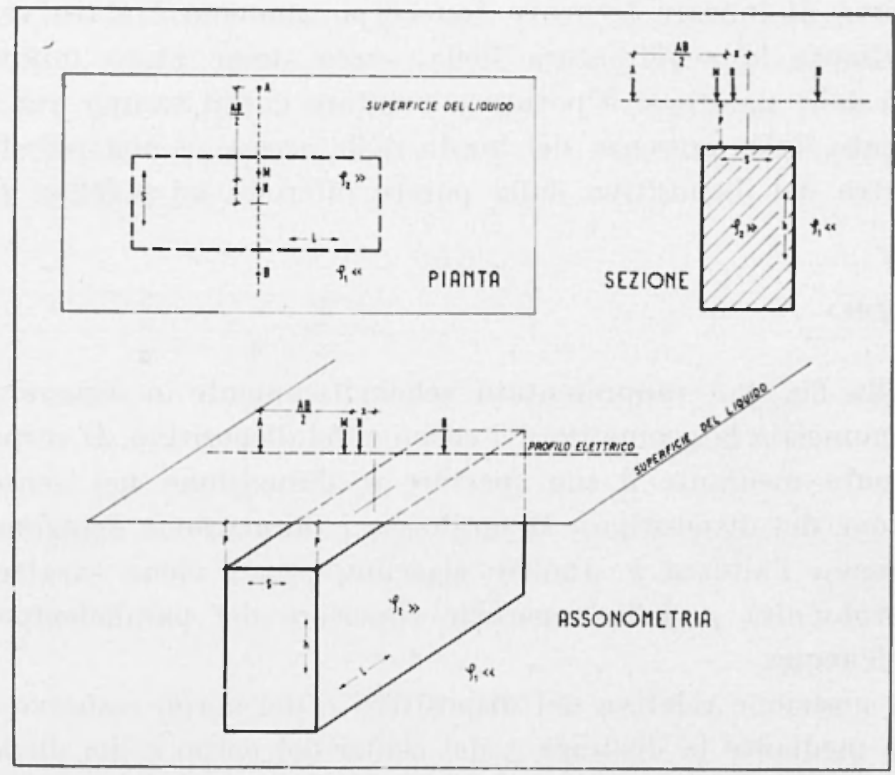

Fig. 1 - Pianta, sezione e assonometria.

gamento della zona in cui è rilevabile l'anomalia. Pertanto, diminuendo il gradiente dell'anomalia, nel caso che i terreni superficiali non siano omogenei nel senso della traslazione del dispositivo, si potrebbero confondere le variazioni laterali di resistività con l'effetto della presenza di una massa anomala o viceversa.

\section{Generalit À}

Le prove su modelli sono state eftettuate con l'apparecchiatura sperimentale dell'Istituto di Geofisica Mineraria già descritta da Grandinetti $\left({ }^{2}\right)$.

È stato impiegato un dispositivo di tipo Schlumberger con elettrodi di corrente di rame el elettrodi di potenziale di platino. La distanza elettrodica $A B$ è stata fissata in $12 \mathrm{~cm}$.

L'acqua che fungeva da mezzo conduttivo era stata resa tale con l'aggiunta di $\mathrm{NaCl}$ in quantità sufficiente a portare la resistività della soluzione attorno ai $3 \mathrm{ohm} \cdot \mathrm{m}$.

Il corpo anomalo ad alta resistività è stato realizzato mediante elementi parallelepipedi di plexiglass tali da permettere, mediante loro combinazione, la realizzazione di corpi di dimensioni diverse. 
Prima di iniziare le prove con corpo anomalo è stato verificato se, mediante la schermatura della vasca, fosse stato minimizzato l'effetto delle pareti; si è potuto constatare che il campo non veniva influenzato dalla presenza del bordo della vasca se non per distanza del centro del dispositivo dalla parete inferiore ad $A B$.

\section{GEOMETRIA}

Nella fig. 1 i rappresentata schematicamente in sezione, pianta ed assonometria la geometria del corpo e del dispositivo. Il corpo viene individuato mediante il suo spessore $s$ (dimensione nel senso della traslazione del dispositivo); la larghezza $l$ (dimensione ortogonale alla traslazione); l'altezza $h$. Inoltre ciascuna prova viene caratterizzata dalla profondità $p$ della superficie superiore del parallelepipedo dal pelo dell'acqua.

La posizione relativa del dispositivo e del corpo resistivo è individuata mediante la distanza $x$ dei centri del corpo e del dispositivo.

Il parallelepipedo resistivo è stato mantenuto, in tutte le prove, ortogonale alla direzione della traslazione e con la superficie superiore parallela alla superficie del liquirlo.

Le resistività apparenti $o_{a}$ misurate sono state riferite alla resistività @1 della soluzione per evitare errori connessi a possibile variazione della resistività della soluzione stessa.

\section{Prove E RISULTATI}

Una prima serie di quattro prove è stata realizzata fissando le dimensioni del corpo $(s=0,500 A B ; \hbar=0,833 A B: l=1,666 A B)$ e facendo variare la profondità $p$ della superficie superiore del parallelepipedo dal pelo dell'acqua. Sono state realizzate prove per $p$ pari a $0,083 A B, 0,125 A B, 0,250 A B, 0,375 A B$. I risultati di queste prove sono riportati nella fig. 2 nella quale in ascisse compare la distanza $X / A B$ del centro del dispositivo dal centro del disturbo e in ordinate il rapporto $\varrho_{a} / \varrho_{1}$.

Successivamente mantenendo costante la profondità $(p=0,125 \mathrm{AB}$ e $p=0,250 A B)$, l'altezza $(h=0,833 A B)$ e la lunghezza $(l=1,666 A B)$ è stato variato lo spessore ( $s$ pari a $0,016 A B, 0,166 A B, 0,333 A B$, $0,500 A B, 1,000 A B, 2,000 A B)$. Si sono cosi ottenute rispettivamente le curve di fig. 3 e 4 per $p 0,125 A B$ e $0,250 A B$. 
E stata fatta poi variare l'altezza $h$ del parallelepipedo $(0,166.4 B$, $0,277 A B, 0,555 . A B, 0,833 A B, 1,666 A B, 3,000 A B)$ mantenendo fissa la profonditì $(p=0,125 A B)$, lo spessore e la larghezzal $(s=0,500 \mathrm{AB}$ e $l-1,666 A B)$. I profili di resistività così ricavati sono stati rappresentati nella fig. 5.

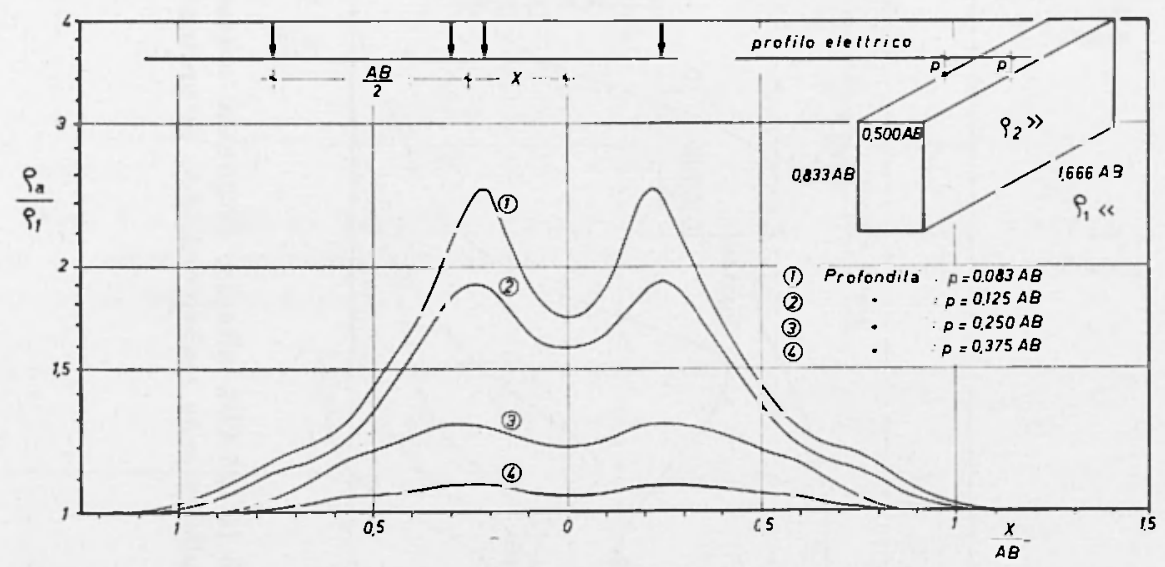

Fír. 2 - Profili di resistività. Profondità $p$ variabile. Spessore, altezza. larghezza costanti. In ascisse la distanza del centro del dispositivo dal centro del parallelepipedo resitivo $(X / A B)$, in ordinata il rapporto fra la resistività apparente $\varrho_{\alpha}$ e la resistività $\varrho_{1}$ della soluzione conduttiva $\varrho_{a} / Q_{1}$.

Infine mantenendo costanti gli altri parametri $(p=0,125 \mathrm{AB}$, $s-0,500 A B, h=0,833 A B)$ sono stati fatti assumere alla lunghezza $l$ seguenti valori: $0,277 A B, 0,5 \tilde{5} 5 A B, 0,833 A B, 1,666 A B, 3,333 A B$. I risultati cosi ottenuti compaiono nella fig. 6 .

Si esaminano ora i profili di resistività ricavati allo scopo di individuare l'influenza dei parametri $p, s, h, l$ sui valori massimi e sull'andamento dei profili di resistività.

La fig. 2 evidenzia la grande influenza della profondità del corpo sui valori massimi dell'anomalia che esso produce. Per meglio individuare questa dipendenza nella fig. 7 sono stati riportati in ordinata i valori massimi dell'anomalia e in ascissa la profondità ovviamente riferita alla distanza elettrodica $A B$. E possibile cosi notare come $\mathrm{i}$ valori massimi aumentino con l'andamento iperbolico verso le profondità più piccole per diminuire altrettanto rapidamente verso le profondità maggiori. Per profonditì maggiori di $0,4-0,5 \quad A B$ l'effetto del parallelepipedo resistivo risulta praticamente trascurabile. 


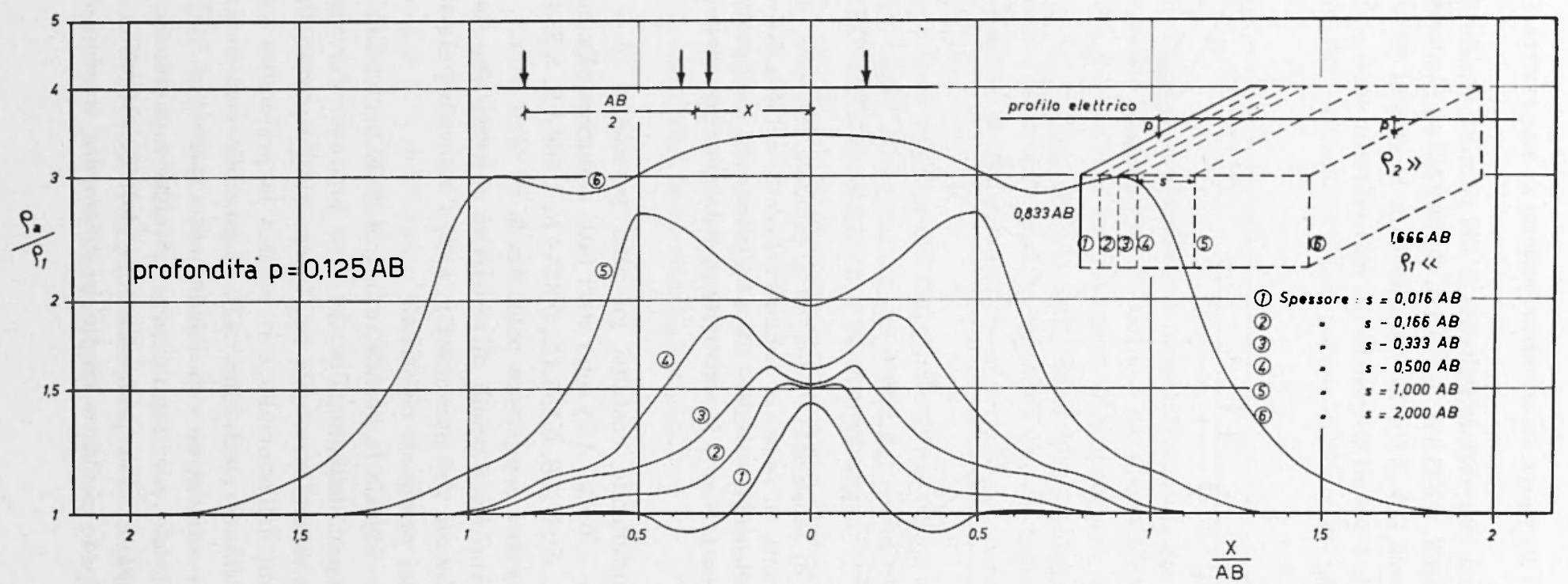

Fig. 3 - Profili di resistività. Spessore $s$ variabile. Profondità $(0,125 A B)$, altezza, larghezza costanti. In ascisse la distanza del centro del dispositivo dal centro del parallelepipedo resistivo $X / A B$, in ordinata il rapportoon/ $\varrho_{2}$. 


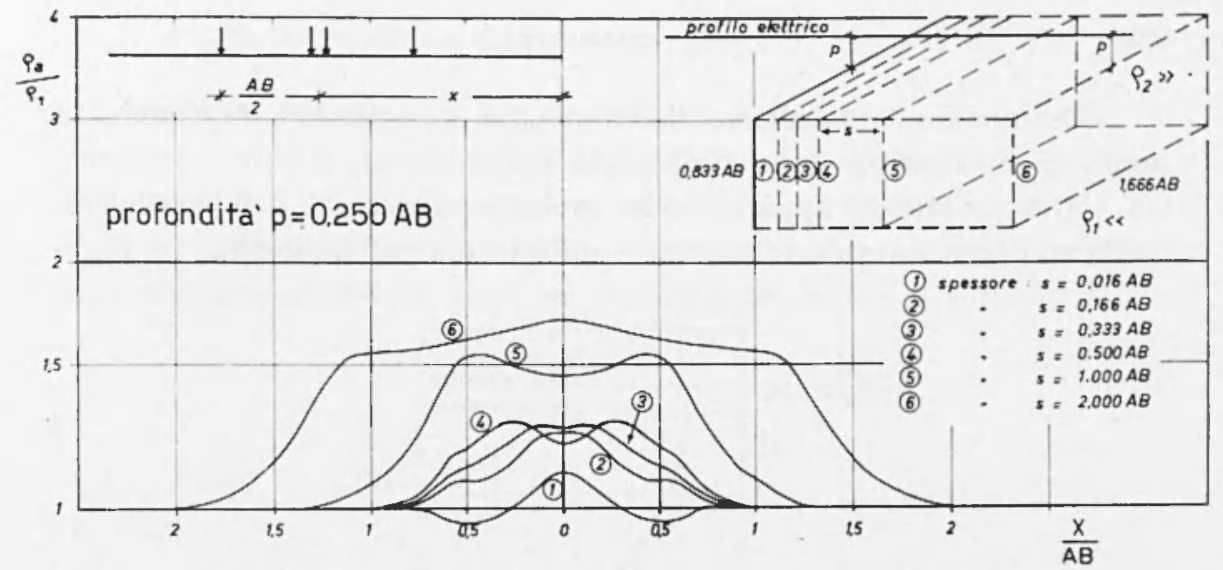

Fig. 4 - Profili di resistivita. Spessore \& variabile. Profondita $(0,250.1 B)$, altezza, larghezza costanti. In ascisse il rapporto $X / A B$, in ordinata il rapporto $\varrho_{a} / \varrho_{1}$.

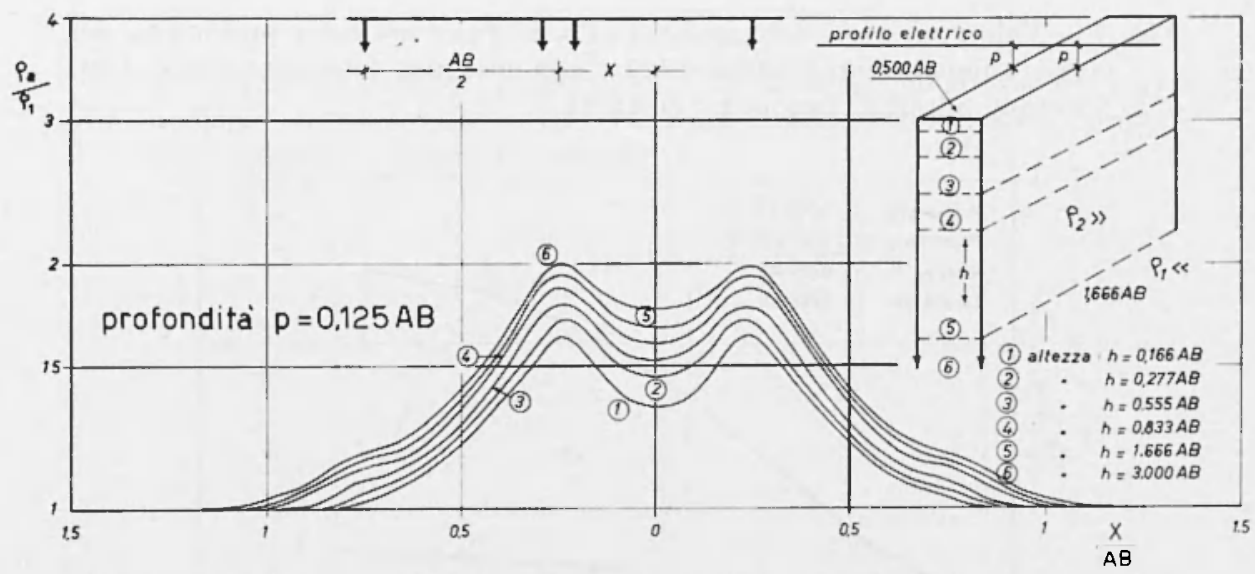

Fig. 5 - Profili di resistivitì. Altezza $h$ variabile. Profonditi, spessore, larghezza costanti. In ascisse il rapporto $X / A B$; in ordinata il rapporto $Q_{a} / Q_{1}$.

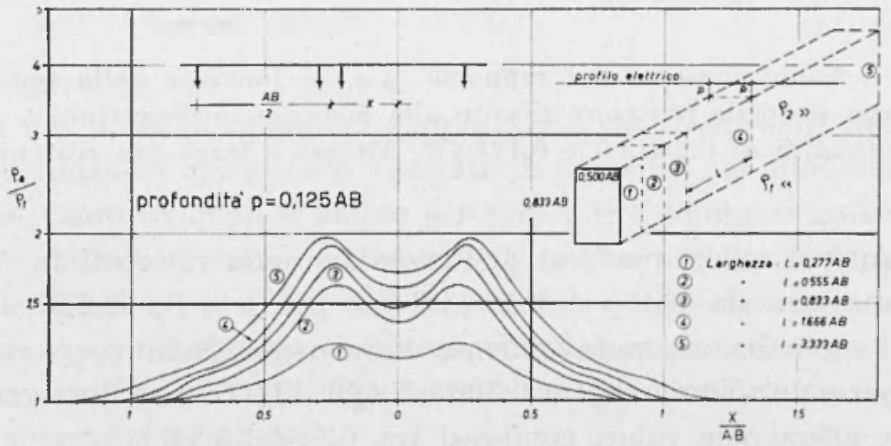

Fig. 6 - Profili di resistiviti. Larghezza $l$ variabile. Profonditi, spessore, altezza costanti. In ascisse il rapporto $X / A B$, in ordinata il rapporto $\varrho_{a} / \varrho_{\text {. }}$. 
Per contro la larghezza dell'anomalia non sembra dipendere in modo reterminante ralla profondità $p$.

Molto influente appare essere anche lo spessore $s$ del parallelepiperlo resistivo sui valori massimi e sulla forma clell'anomalia. La fig. 8

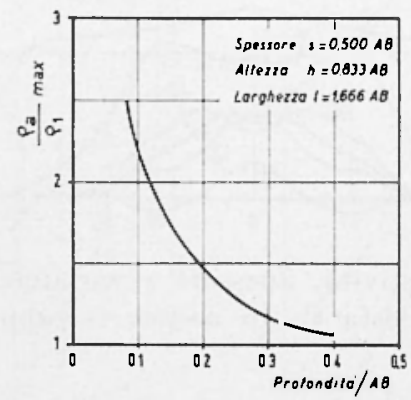

Fig. 7 - Valori massimi del rapporto $\varrho_{a} / \varrho_{1}$ in funzione della profondita del corpo anomalo (profonditì riferita alla distanza interelettrodica $A B$ ). spessore, altezza, larghezza costanti.

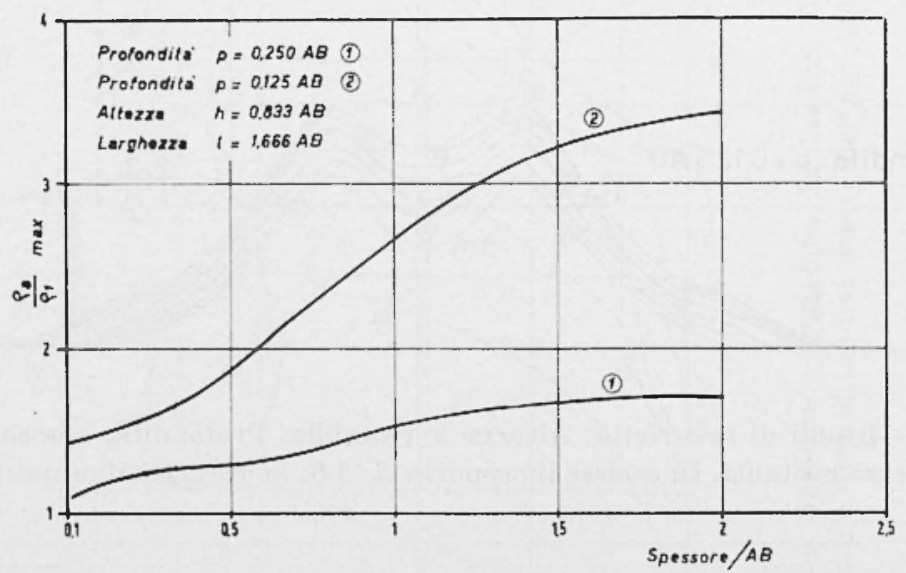

Tig. 8 - Valori massimi del rapporto $\varrho_{a} / \varrho_{1}$ in funzione dello spessore del corpo anomalo (spessore riferito alla distanza interelettrodica $A B$ ) per profondità di $0,250.1 B$ e $0,125 A B$. Altezza e larghezza costanti.

nella quale i valori massimi rlell'anomalia sono riportati in funzione dello spessore sia per $p=0,125 A B$ sia per $p=0,250 A B$, dimostra come l'anomalia sia notevolmente influenzata dallo spessore sopratutto per valori compresi tra 0,300 e 2,000 $A B$. I a massima variazione sembra aversi per valori compresi tra 0,5 e $1,5 A B$. 
Inoltre, a differenza di quanto avveniva per la profondità, lo spessore influenza grandemente la distanza a cui si risente la presenza del corpo resistivo, come appare dalle figure 3 e 4 . Ad esempio nel caso $p=0,250 A B$ e $s=2,000 A B$ l'anomalia assume valori inferiori agli errori sperimentali solo per le distanze superiori a $2 A B$.

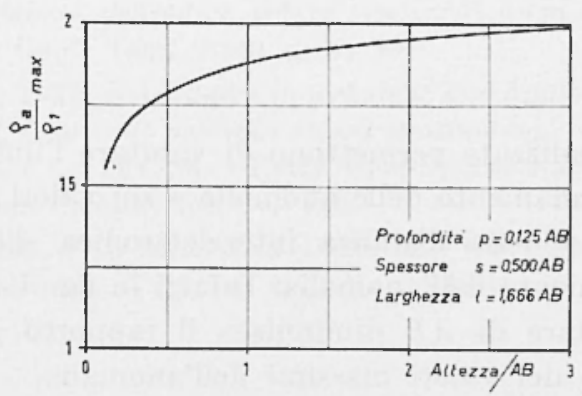

Fig. 9 - Valori massimi del rapporto $g_{a} \varrho_{1}$ in funzione dell'altezza del corpo anomalo (altezza riferita alla distanza interelettrodica $A B$ ). Profonditi. spessore, larghezza costanti.

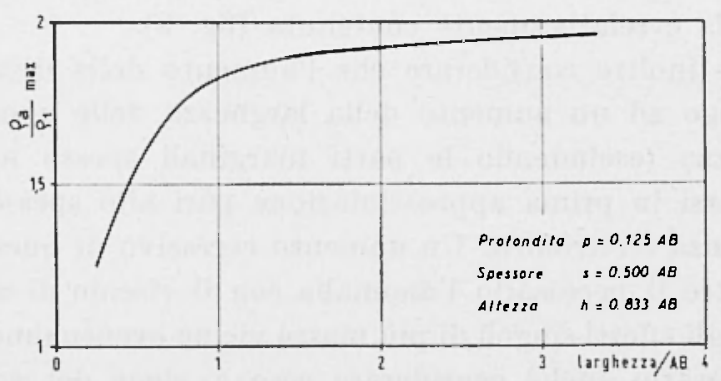

Fig. 10 - Valori massimi del rapporto $Q_{\alpha} / \varrho_{1}$ in funzione della larghezza del corpo anomalo (larghezza riferita alla distanza interelettrodica $A B$ ). I'rofonditi, spessore, altezza costanti.

La fig. 9, nella quale sono riportati i valori massimi delle anomalie in funzione dell'altezza $h$, mette in evidenza che modeste variazioni dei valori anomali si hanno per valori di $h$ compresi grosso modo tra 0,3 e $0,8 A B$. Il valore dell'anomalia risulta praticamente indipendente dall'altezza per valori di $h$ maggiori di $0,8 A B$ mentre per valori inferiori a $0,3 A B$ l'anomalia decresce rapidamente.

Come risulta poi dalla fig. 5 l'altezza non modifica praticamente la larghezza dell'anomalia. 
Per valori di $l$ inferiori a $0,5, A B$ l'anomalia aumenta rapidamente con $l$; tale aumento risulta meno notevole per valori di $l$ compresi tra 0,5 e 1,5 , per divenire molto modesto per valori di $l$ superiori a 1,5 (fig. 10).

Anche in questo caso, come per l'altezza, l'aumento della larghezza (fig. (i) amplia molto poco l'area nella quale è possibile misurare l'anomalia.

\section{Concitisioni}

Le prove realizzate permettono di valutare l'influenza dei singoli parametri sull'andamento delle anomalie e sui valori massimi.

Un aumento della distanza interelettrodica $A B$ provoca, come previsto, un aumento dell'anomalia. Infatti in fig. 7 è possibile notare come all'aumentare di $A B$ diminuisca il rapporto $p / A B$ con conseguente aumento dei valori massimi dell'anomalia.

I'altra parte però dalla fig. 8 si può rilevare come un aumento della distanza $A B$ provochi a parità di spessore una diminuzione della anomalia. Se però il rapporto $s / A B$ resta superiore a 0,5 (per $p=0,250 A B$ ) ovvero ad 1 (per $p=0,125 . A B$ ) la diminuzione dell'anomalia è relativamente contenuta (fig. 8).

Occorre inoltre considerare che l'aumento della distanza elettrodica dì luogo arl un aumento della larghezza delle anomalie: infatti tale larghezza (escludendo le parti marginali spesso non rilevabili) può assumersi in prima approssimazione pari allo spessore del corpo più la distanza elettrodica. Un aumento eccessivo di quest'ultima puó allargare oltre il necessario l'anomalia con il rischio di non riuscire a distinguere gli effetti singoli di più masse vicine eventualmente presenti.

$\grave{E}$ necessario anche considerare cosa avviene dei valori massimi dell'anomalia, quando, aumentando la distanza elettrodica $A B$, diminuiscono i rapporti $h / A B$ (fig. 9) e $l / A B$ (fig. 10). In entrambi i casi l'anomalia decresce al diminuire del rapporto: per l'altezza è però sufficiente mantenere valori superiori o uguali a 0,3 per non provocare una brusca diminuzione dell'anomalia; per la larghezza, per contro, il rapporto non deve scendere al di sotto di 0,6 . L'influenza dell'altezza e della larghezza diviene trascurabile quando si superino rispettivamente i limiti di $0,7 A B$ e $1,5 A B$.

Si deve quindi concludere che nella scelta della distanza interelettrodica bisogna realizzare il migliore compromesso tra le influenze contrastanti della profondità da una parte e dello spessore altezza e larghezza dall'altra (con maggior riguardo per lo spessore). 


\section{BIBIUOGRAFIA}

(1) GoUdswaRD W., 1957. - On the effect of the Tank wall material in geoelectrical model experiments. "Geoph. Prospecting ", V, 272.

(2) Graxdinetti II., 1968. - Risultati di prove su modelli relative ad una muova disposizione elettrodica per la ricerca di corpi di limilate dimensioni. "Boll. Geof. Teor. Appl. ", X, 39.

(3) HenLAN C. A., 1932. - Advances in technique and application of resistivity and potential drop ratio methods in oil prospecting. "Geophysies ", III, 90. Ristampato nel 1947 in: "Early Geophysical Papers of the Society. of Exploration Geophysicists", 420.

(4) SARtori R., 1955. - La vasca elettrolitica. Atti Convegno Interno. "I 110 delli nella tecnica n, Venezia, Acc. Naz. Lincei. 